\title{
¿Puede la contaminación orgánica afectar la tasa de crecimiento de los octocorales en el Caribe?
}

\section{Can organic pollution affect the growth rate of octocorals in the Caribbean?}

\author{
Néstor Rey-Villiers ${ }^{1,2 *}$ \& Alberto Sánchez ${ }^{1}$ \\ ${ }^{1}$ Centro Interdisciplinario de Ciencias Marinas, Instituto Politécnico Nacional. Av. IPN S/N, Col. Playa Palo de Sta. Rita, Apdo. \\ Postal \#592, 23096, La Paz, Baja California Sur, México. \\ ${ }^{2}$ Instituto de Ciencias del Mar, Ministerio de Ciencia, Tecnología y Medio Ambiente (CITMA). Loma y 39, Plaza, CP: 10600, \\ La Habana, Cuba. \\ *Email: nestorrvilliers@gmail.com
}

\begin{abstract}
RESUMEN
La contaminación orgánica en el litoral de La Habana está afectando las asociaciones de octocorales, y poco se conoce su efecto en la tasa de crecimiento (TC). Se empleó un transecto de banda fijo $(250 \times 2 \mathrm{~m})$ para localizar las colonias. La TC de Eunicea flexuosa y Plexaura kuekenthali fue significativamente mayor en el arrecife con más contaminación orgánica (3,6 cm/año y 4,1 cm/año, respectivamente) vs. sitios de referencia, lo que parece estar relacionado con su heterotrofía.
\end{abstract}

\begin{abstract}
Organic pollution in the Havana coastline is affecting octocorals assemblages, and little is known about its effect on the growth rate (GR). A fixed belt transect $(250 \times 2 \mathrm{~m})$ was used to locate the colonies. The GR of Eunicea flexuosa and Plexaura kuekenthali was significantly higher in the reef with more organic pollution $(3,6 \mathrm{~cm} / \mathrm{año}$ y $4,1 \mathrm{~cm} / \mathrm{año}$, respectively) vs. reference sites, which seems to be related to their heterotrophy.
\end{abstract}

Los octocorales son el segundo grupo más común de los animales macrobentónicos sésiles en los arrecifes del Caribe y del Indo-Pacífico (Fabricius 2011a), y presentan una gran importancia estructural y funcional en este ecosistema. El crecimiento de estos organismos se caracteriza por la replicación iterativa de las ramas y los pólipos, y varía de manera intraespecífica e interespecífica (Goffredo \& Lasker 2006; Calixto-Botía \& Sánchez 2017). Los factores ambientales que pueden afectar la tasa de crecimiento de los octocorales son: el flujo del agua, la profundidad, la disponibilidad de luz, la sedimentación, el suministro de alimentos, la temperatura, la depredación, la abrasión y el sobrecrecimiento de organismos (Yoshioka \& Yoshioka 1991; Goh \& Chou 1995; Sánchez et al. 2007; Rossi et al. 2011).

El crecimiento acelerado de la población humana en áreas costeras ha provocado el incremento de nutrientes en los ecosistemas marinos costeros (Fabricius 2011b). El enriquecimiento de nutrientes en los arrecifes de coral ha traído como consecuencia una disminución en la abundancia, cobertura, diversidad y riqueza de especies de octocorales (Yeung et al. 2014; Rowley 2018), así como cambios fisiológicos en los corales pétreos y afectaciones en la estructura de la comunidad arrecifal (Fabricius et al. 2012; Baum et al. 2015). La región noroccidental de Cuba no está exenta de esta problemática, ya que los arrecifes del litoral de La Habana son afectados por la contaminación orgánica proveniente de las descargas residuales a través de ríos, difusores submarinos y vertimientos costeros. Las consecuencias de la contaminación orgánica en los arrecifes de La Habana son la perdida de diversidad y abundancia de octocorales (Hernández-Muñoz et al. 2008; Rey-Villiers 2009), reducción en la cobertura de corales pétreos (Durán et al. 2018; González-Díaz et al. 2018) y proliferación de macroalgas (Durán et al. 2018). Además, la contaminación orgánica disminuye la altura de los octocorales Eunicea flexuosa (Lamouroux, 1821) y Plexaura kuekenthali Moser, 1921, probablemente, por un aumento en la mortalidad o por una reducción en la tasa de crecimiento (Rey-Villiers $\&$ Alcolado 2012). Sin embargo, se conoce muy poco sobre cómo la contaminación orgánica puede afectar la tasa de crecimiento de los octocorales, y al parecer, el crecimiento de estos organismos es más rápido ante el aporte de nutrientes (Aliño et al. 1992) y microorganismos 
(Manrique-Rodríguez et al. 2006). En los arrecifes de La Habana predominan los octocorales E. flexuosa y $P$. kuekenthali, y son consideradas especies tolerantes a la contaminación orgánica (Hernández-Muñoz et al. 2008; Rey-Villiers 2009), por lo que la tasa de crecimiento de estas especies podría aumentar con el aporte de nutrientes, materia orgánica y microorganismos. El objetivo es estimar la tasa de crecimiento anual de E. flexuosa y P. kuekenthali en tres arrecifes coralinos del Caribe con diferentes condiciones de contaminación orgánica.

La investigación se realizó en tres arrecifes al noroeste de Cuba, a $10 \mathrm{~m}$ de profundidad y con diferente distancia a la Bahía de La Habana. El sitio Parque Antonio Maceo (PAM) se localiza a una distancia aproximada de 1,4 Km al oeste de la Bahía de La Habana, y los sitios Playa del Salado (Sa) y Boca de Calderas (Ca) se ubican entre 30 y $35 \mathrm{Km}$ al oeste y este, respectivamente de esta bahía (Fig. 1). Se consideró al sitio PAM como el de mayor contaminación orgánica por la elevada concentración de esteroles fecales e hidrocarbonos alifáticos en los sedimentos superficiales (Castellanos-Iglesia et al. 2018; Martins et al. 2018), y por su cercanía a la Bahía de la Habana, la cual vierte grandes cantidades de materia orgánica y nutrientes (Beltrán et al. 2012). Además, los municipios más cercanos a esta bahía presentan una elevada densidad poblacional, que son los municipios Habana Vieja y Centro Habana con 19679 y 40710 habitantes $/ \mathrm{Km}^{2}$, respectivamente (ONE 2016). Los sitios $\mathrm{Sa}$ y $\mathrm{Ca}$ se emplearon como referencia por estar alejados de la Bahía de La Habana, y por presentar el tejido de la macroalga Sargassum hystrix J. Agardh en Ca un $\delta^{15} \mathrm{~N}$ menor de $2 \%$, y con el menor contenido de nitrógeno entre los arrecifes del norte de La Habana (Durán et al. 2018).
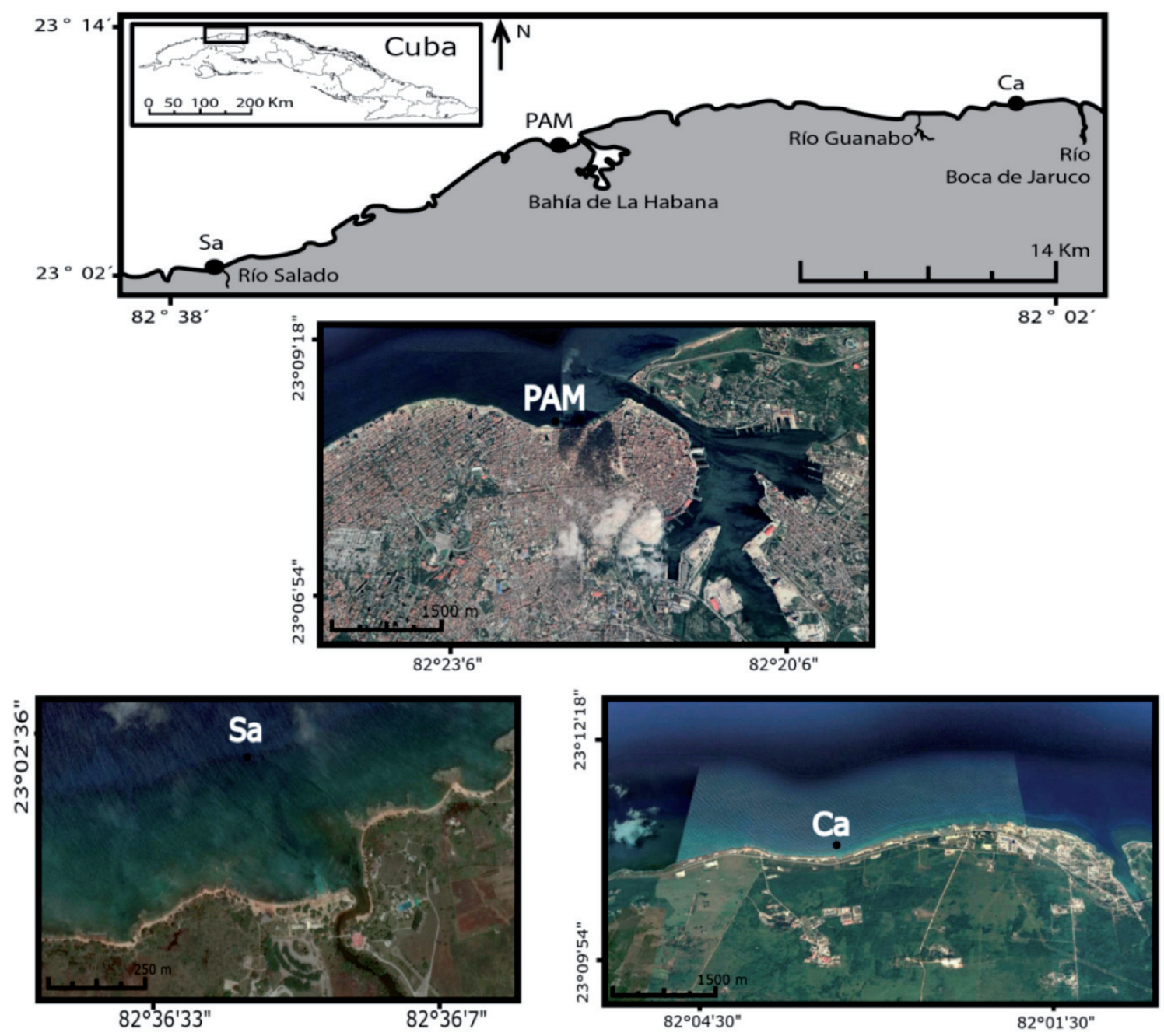

Figura 1: Mapa de los sitios de muestreos y las cuencas fluviales cercanas. Sa: Playa del Salado, PAM: Parque Antonio Maceo, Ca: Boca de Calderas. / Map of sampling sites and nearby river basins. Sa: Playa del Salado, PAM: Parque Antonio Maceo, Ca: Boca de Calderas. 
Las colonias de E. flexuosa y P. kuekenthali fueron marcadas inicialmente en abril de 2011 y revisitadas en abril de 2012. Las marcas consistieron en una chapilla enumerada atada a la base de las colonias. El número de colonias marcadas al inicio varió en dependencia de la densidad de cada especie. En Sa se marcaron 66 colonias de E. flexuosa y 76 de $P$. kuekenthali, en Ca 75 colonias de E. flexuosa y 49 de $P$. kuekenthali y en PAM se marcaron 49 colonias de $E$. flexuosa y 63 de P. kuekenthali.

Se empleó un transecto de banda fijo de $250 \mathrm{~m}$ de largo por $1 \mathrm{~m}$ de ancho haciendo un recorrido de ida y vuelta a cada lado de la cinta $(250 \mathrm{~m} \times 2 \mathrm{~m})$ donde se localizaron las colonias. Se realizó un mapa de posición de las colonias que consistió en anotar a que distancia del inicio de la cinta métrica se localizaba cada una y si estaban a la izquierda o derecha de la cinta. Inicialmente, se marcaron colonias entre 10 y $20 \mathrm{~cm}$ de altura para evitar medir colonias de diferentes intervalos de tallas, ya que la tasa de crecimiento de los octocorales disminuye con su altura (Lasker et al. 2003; Goffredo \& Lasker 2006). La altura desde la base de las colonias hasta el extremo de sus ramas se midió al inicio del estudio y un año después para determinar la tasa de crecimiento. Las mediciones se realizaron con una cinta métrica con un nivel de precisión de $\pm 0,1 \mathrm{~cm}$.

Se tomaron muestras de agua con un frasco de vidrio estéril de $150 \mathrm{~mL}$ para determinar las concentraciones de bacterias coliformes totales (CT) y fecales (CF), estreptococos fecales (EF), bacterias sulfatoreductoras (SR) y heterótrofas $(\mathrm{BH})$. Las concentraciones de CT, CF, EF y SR se determinaron a partir de la metodología de diluciones seriadas en tubos múltiples (APHA 1998), y se cuantificaron a partir de la Tabla de Número Más Probable de APHA(1998). La concentración de $\mathrm{BH}$ se determinó a través del método de siembra en superficie descrito en Miravet et al. (2009). También se tomaron muestras de agua en frascos plásticos de $1 \mathrm{~L}$ para determinar las concentraciones de $\mathrm{NH}_{4}^{+}, \mathrm{NO}_{3}$, $\mathrm{NO}_{2}^{-}$y $\mathrm{PO}_{4}{ }^{3-}$ a partir de la metodología propuesta en $\mathrm{FAO}$ (1975) e IOC-UNESCO (1983). La visibilidad horizontal del agua se midió con un disco Secchi a $0,5 \mathrm{~m}$ por debajo de la superficie (Rogers et al. 2001).

La tasa de crecimiento de las dos especies se obtuvo restando la medición de la altura final a la inicial de las colonias inspeccionadas durante un año. Se construyeron dos matrices de similitud con la tasa de crecimiento de $E$. flexuosa y $P$. kuekenthali a partir de la distancia euclidiana. La tasa de crecimiento de estas especies se comparó entre sitios para evaluar el efecto de la contaminación de la Bahía de La Habana a partir de un análisis de varianza univariado y unifactorial en base a 999 permutaciones sin restricciones de los datos crudos (PERMANOVA) (Anderson et al. 2008). Se calculó el 95\% del intervalo de confianza $(95 \%$ IC) a partir de la prueba de Monte Carlo con 10000 iteraciones y por el método de los percentiles. El solapamiento de los intervalos de confianza fue empleado como criterio de no diferencias significativas entre pares de medias, y se calculó el tamaño de efecto y el 95\% IC entre los pares de medias que tuvieron diferencias significativas. La tasa de crecimiento fue relacionada con las variables microbiológicas y físico-químicas a partir del coeficiente de correlación de Pearson. La matriz de pares de valores se construyó con la tasa de crecimiento de E. flexuosa y $P$. kuekenthali por sitios y las variables microbiológicas y físico-químicas. Para determinar la probabilidad de que por azar los pares de variables se correlacionen, se construyó un modelo nulo con la simulación de Monte Carlo con 10000 iteraciones. Se consideró 0,05 como nivel de significación.

La tasa de crecimiento de E. flexuosa varió de $0,1 \mathrm{~cm} /$ año en los sitios $\mathrm{Sa}$ y $\mathrm{Ca}$ a $7,7 \mathrm{~cm} /$ año en PAM. La tasa de crecimiento de esta especie en PAM (3,6 cm/año 95\% IC: $2,9-4,3 \mathrm{~cm} / \mathrm{año}$ ) fue significativamente superior en comparación con Sa (1,7 cm/año 95\% IC: 1,4-2,1 cm/año) y $\mathrm{Ca}(1,8 \mathrm{~cm} /$ año $95 \%$ IC: 1,5-2,2 cm/año) (PERMANOVA, pseudo-F $\left._{(2 ; 105)}=18,4 ; \mathrm{P}=0,001\right)$ (Fig. 2a). El tamaño de efecto entre PAM y Sa fue 1,9 cm/año (95\% IC: $1,1-2,7 \mathrm{~cm} /$ año) y entre PAM y Ca fue 1,8 cm/año (95\% IC: 1,0-2,6 $\mathrm{cm} / \mathrm{año}$ ), lo que representa que como promedio las colonias de E. flexuosa crecen aproximadamente $2 \mathrm{~cm} /$ año más rápido en PAM que en Sa y Ca. En $P$. kuekenthali, la tasa de crecimiento fluctuó entre $0,1 \mathrm{~cm} /$ año en $\mathrm{Sa}$ y Ca hasta $9,4 \mathrm{~cm} /$ año en PAM. La tasa de crecimiento de esta especie en PAM (4,1 cm/año 95\% IC: 3,1-5,0 cm/año), también fue significativamente mayor en comparación con $\mathrm{Sa}(1,8 \mathrm{~cm} /$ año $95 \%$ IC: $1,3-2,4 \mathrm{~cm} /$ año $)$ y Ca $(1,1 \mathrm{~cm} /$ año $95 \%$ IC: 0,7-1,5 cm/año) (PERMANOVA, pseudo-F ${ }_{(2 ; 59)}=14,9 ; \mathrm{P}$ $=0,001)$ (Fig. 2b). El tamaño de efecto entre PAM y Ca fue $3 \mathrm{~cm} /$ año ( $95 \%$ IC: $2,7-3,3 \mathrm{~cm} / \mathrm{año}$ ) y entre PAM y Sa fue 2,3 cm/año (95\% IC: $1,1-3,3 \mathrm{~cm} / \mathrm{año}$ ), lo que evidencia que las colonias de $P$. kuekenthali como promedio crecen 3 $\mathrm{cm} /$ año y $2,3 \mathrm{~cm} /$ año más rápido en PAM que en $\mathrm{Ca}$ y $\mathrm{Sa}$, respectivamente.

Estos resultados evidencian que E. flexuosa y $P$. kuekenthali están creciendo más rápido en condiciones de mayor concentración de materia orgánica y nutrientes aportados por la Bahía de La Habana. La tasa de crecimiento de ambas especies presentó una correlación positiva con los $\mathrm{CF}, \mathrm{CT}, \mathrm{BH}, \mathrm{SR}, \mathrm{EF}, \mathrm{NH}_{4}^{+}, \mathrm{PO}_{4}^{3-}$, y una correlación negativa con la visibilidad del agua y la concentración de $\mathrm{NO}_{3}^{-}+\mathrm{NO}_{2}^{-}$(Tabla 1). Esto sugiere que en condiciones de aguas enriquecidas en materia orgánica, microorganismos y nutrientes como en PAM, se acelera la tasa de crecimiento de E. flexuosa y $P$. kuekenthali, y es posible que la heterotrofía de estas especies constituya una fuente de energía adicional a la obtenida por fotosíntesis para su crecimiento. En correspondencia con esto, la eficiencia fotosintética de los octocorales es generalmente baja, con ganancia de carbono excediendo la respiración solamente en elevados niveles de luz (Fabricius \& Klumpp 1995). El sitio PAM presentó la visibilidad del agua más baja entre los arrecifes del norte de 
La Habana $(11,8 \mathrm{~m} \pm 5,0 \mathrm{~m})$, por lo tanto, es probable que una parte de la energía que emplean en el crecimiento sea obtenida por la heterotrofía de las colonias. Los octocorales tienen la capacidad de consumir microorganismos del plancton (Ribes et al. 1998; Coma et al. 2015). Ribes et al. (1998) señalan que E. flexuosa es capaz de capturar presas del microplancton como ciliados, dinoflagelados y diatomeas. Por su parte, Coma et al. (2015) reportan que el octocoral zooxantelado Eunicella singularis (Esper, 1791) captura zooplancton entre 40 y $920 \mu \mathrm{m}$.

Los resultados en el crecimiento de E. flexuosa y $P$. kuekenthali en PAM coinciden con otras investigaciones realizadas en octocorales (Aliño et al. 1992; ManriqueRodríguez et al. 2006). Aliño et al. (1992) señalan que la tasa de crecimiento en el octocoral Clavularia inflata Schenk, 1896 aumentó en un arrecife cerca de la costa con mayores niveles de nutrientes en la Gran Barrera de Coral de Australia. Por su parte, Manrique-Rodríguez et al. (2006) plantean que la elevada tasa de crecimiento de Gorgonia ventalina Linnaeus, 1758 puede estar determinada por la disponibilidad de partículas suspendidas y microorganismos del plancton, proporcionados por la escorrentía y aguas residuales en la bahía de Santa Marta, Colombia.

Los resultados evidencian que la tasa de crecimiento de E. flexuosa y P. kuekenthali aumenta con un mayor aporte de materia orgánica, microorganismos y nutrientes.
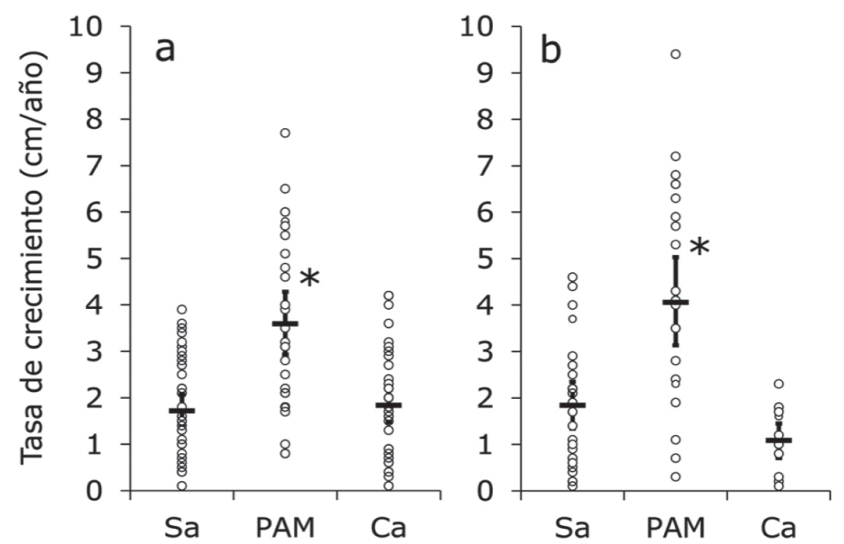

Figura 2: Tasa de crecimiento anual (media, 95\% IC y valores observados) de E. flexuosa (a) y P. kuekenthali (b) en tres arrecifes de la región noroccidental de Cuba. Sa: Playa del Salado, PAM: Parque Antonio Maceo, Ca: Boca de Calderas. * Indica significación estadística entre los sitios con el PERMANOVA. / Annual growth rate (mean, 95\% CI and observed values) of E. flexuosa (a) and $P$. kuekenthali (b) in three reefs of the northwestern region of Cuba. Sa: Playa del Salado, PAM: Parque Antonio Maceo, Ca: Boca de Calderas. * Indicate statistical significance among sites with PERMANOVA.

Tabla 1: Coeficiente de correlación de Pearson entre la tasa de crecimiento (TC) de E. flexuosa y P. kuekenthali y las variables microbiológicas y físico-químicas. El número entre paréntesis indica la probabilidad (P). CF: bacterias coliformes fecales, CT: bacterias coliformes totales, BH: bacterias heterótrofas, SR: bacterias sulfato-reductoras, EF: estreptococos fecales, Vis: visibilidad horizontal del agua. / Pearson correlation coefficient between the growth rate (GR) of E. flexuosa and P. kuekenthali and the microbiological and physicochemical variables. The number in parentheses indicates the probability (P). CF: fecal coliform bacteria, CT: total coliform bacteria, BH: heterotrophic bacteria, SR: sulfate-reducing bacteria, EF: fecal streptococci, Vis: horizontal visibility of water.

\begin{tabular}{lccccccccc}
\hline Variables & $\mathrm{CF}$ & $\mathrm{CT}$ & $\mathrm{BH}$ & $\mathrm{SR}$ & $\mathrm{EF}$ & $\mathrm{Vis}$ & $\mathrm{NH}_{4}^{+}$ & $\mathrm{NO}_{3}^{-}+\mathrm{NO}_{2}^{-}$ & $\mathrm{PO}_{4}^{3-}$ \\
\hline TC de E. flexuosa & 0,96 & 0,96 & 0,95 & 0,96 & 0,96 & $-0,94$ & 0,97 & $-0,82$ & 0,95 \\
y P. kuekenthali & $(0,04)$ & $(0,04)$ & $(0,01)$ & $(0,01)$ & $(0)$ & $(0,04)$ & $(0,009)$ & $(0,04)$ & $(0,04)$ \\
\hline
\end{tabular}

\section{AGRADECIMIENTOS}

Al Dr. Pedro Alcolado por su incondicional guía y al MSc. Pedro Alcolado Prieto y los buzos Johannes Acosta, Jorge Oliva y Sandy León por el apoyo en los muestreos.

\section{REFERENCIAS}

Aliño, P.M., Sammarco, P.W., Coll, J.C. 1992. Competitive strategies in soft corals (Coelenterata, Octocorallia). IV. Environmentally induced reversals in competitive superiority. Marine Ecology Progress Series 81: 129-145.

ApHA 1998. Standard Methods for Examination of Water and
Wastewater. 20th. ed., Washington D.C. American Public Health Association. 210 pp.

Anderson, M.J., Gorley, R.N., Clarke, K.R. 2008. PERMANOVA + for PRIMER: Guide to Software and Statistical Methods. PRIMER-E: Plymounth, UK.

Baum, G., Januar, H.I., Ferse, S.C.A., Kunzmann, A. 2015. Local and Regional Impacts of Pollution on Coral Reefs along the Thousand Islands North of the Megacity Jakarta, Indonesia. PLoS ONE 10(9): 1-26.

Beltrán, J., Pérez, M., Gómez, Y., Regadera, R. 2012. Control y evolución de la calidad ambiental de la Bahía de La Habana y el litoral adyacente. PNAP/GTE. Informe final. Cimab. La Habana, 62 pp.

Calixto-Botía, I., SÁnchez, J.A. 2017. A case of modular phenotypic plasticity in the depth gradient for the gorgonian 
coral Antillogorgia bipinnata (Cnidaria: Octocorallia). BMC Evolutionary Biology 17(1): 1-8.

Castellanos-Iglesias, S., Caroline, A.C., Martins, C.C., Di Domenico, M., Rocha, R.M., Haddad, M.A. 2018. Organic contamination as a driver of structural changes of hydroid's assemblages of the coral reefs near to Havana Harbour, Cuba. Marine Pollution Bulletin 133: 568-577.

Coma, R., Llorente-Llurba, E., Serrano, E., Gili, J.M., Ribes, M. 2015. Natural heterotrophic feeding by a temperate octocoral with symbiotic zooxanthellae: a contribution to understanding the mechanisms of die-off events. Coral Reef 34: 549-560.

Durán, A., Shantz, A.A., Burkepile, D.E., Collado-Vides, L., Ferrer, V.M., Palma, L., Ramos, A., Gonzalez-Díaz, S. P. 2018. Fishing, pollution, climate change, and the longterm decline of coral reefs off Havana, Cuba. Bulletin of Marine Science 94(2): 213-228.

FABRICIUS, K.E., KLUMPP, D.W. 1995. Widespread mixotrophy in reef inhabiting soft corals: the influence of depth, and colony expansion and contraction on photosynthesis. Marine Ecology Progress Series 125: 195-204.

Fabricius, K. 2011A. Octocorallia. In: Hopley, D. (Ed.). Encyclopedia of Earth Sciencies Series. Encyclopedia of Modern Coral Reefs. Structure, Form and Process, pp. 740-745. James Cook University, Townsville, Australia.

FABRICIUS, K. 2011B. Factors Determining the Resilience of Coral Reefs to Eutrophication: A Review and Conceptual Model. In: Dubinsky, Z. and Stambler, N. (Eds.). Coral Reefs: An Ecosystem in Transition, pp. 493-505. The Mina and Everard Goodman Faculty, Israel.

Fabricius, K., Cooper, T.F., Humphrey, C., Uthicke, S., De’ath, G., Davidson, J., LeGrand, H., Thompson, A., Schaffelke, B. 2012. A bioindicator system for water quality on inshore coral reefs of the Great Barrier Reef. Marine Pollution Bulletin 65: 320-332.

FAo 1975. Manual of Methods in Aquatic Environmetal Research part-1. Methods for detection and monitoring of water pollution. FAO Fish. Tech. Paper. 137: 237 pp.

GofFredo, S., LASKER, H.R. 2006. Modular growth of a gorgonian coral can generate predictable patterns of colony growth. Journal of Experimental Marine Biology and Ecology 336: 221-229.

GoH, N.K.C., Chou, L.M. 1995. Growth of five species of gorgonians (Subclass: Octocorallia) in the sedimented waters of Singapore. Marine Ecology 16(4): 337-346.

González-Díaz, P., González-Sansón, G., Aguilar, C.B., Álvarez, S.F., Perera, O.P., Hernández, L.F., Ferrer, V.M.R., Cabrales, Y.C., Armenteros, M.A., Guardia, E. 2018. Status of Cuban coral reefs. Bulletin of Marine Science 94(2): 229-247.

Hernández-Muñoz, D., Alcolado, P.M., Hernández-González, M. 2008. Efecto de un emisario submarino de residuos urbanos sobre comunidades de octocorales (Octocorallia: Alcyonacea) en Cuba. Revista Biología Tropical 56(1): 65-75.

Ioc-Unesco 1983. Chemical methods for use in marine environmental monitoring. Manual and guides, No. 12.

Lasker, H.R., Boller, M.L., Castanaro, J., Sánchez, J.A. 2003.
Determinate Growth and Modularity in a Gorgonian Octocoral. Biological Bulletin 205: 319-330.

Manrique-Rodríguez, N., Bejarano-Chavarro, S., GarzónFerreira, J.2006. Crecimiento del abanico de mar Gorgonia ventalina (Linnaeus, 1758) (Cnidaria: Gorgoniidae) en el área de Santa Marta, Caribe Colombiano. Boletín de Investigaciones Marinas y Costeras 35: 77-90.

Martins, C.C., Castellanos-Iglesias, S., Caroline, A.C., Câmara de Souza, A., Aliceda, M.F., Pereira, T.A. 2018. Hydrocarbon and sewage contamination near fringing reefs along the west coast of Havana, Cuba: A multiple sedimentary molecular marker approach. Marine Pollution Bulletin 136: 38-49.

Miravet, M.E., Lugioyo, M., Loza, S., Lavandera, D.E., Delgado, Y., Carmenate, M., Pérez, D.M. 2009. Procedimientos para el monitoreo de la calidad ambiental en la zona marino costera a partir de microorganismos. Departamento de Microbiología-Necton, Instituto de Oceanología, CITMA, Cuba, La Habana. Proyecto PNUD/GEF. 95 pp.

OnE, Oficina Nacional de Estadísticas de Cuba. 2016. Anuario Estadístico de Cuba 2015. Población [En línea]. La Habana, Cuba. Accedido: Febrero, 2018. Disponible desde: http://www.one.cu

Rey-Villiers, N. 2009. Características de las comunidades de octocorales y ensayo de su uso como bioindicadores en un gradiente de contaminación en el oeste de La Ciudad de La Habana, Cuba. Tesis de Diploma. Universidad de La Habana, Ciudad Habana, Cuba. 69 pp.

Rey-Villiers, N., Alcolado, P.M. 2012. Variación de la talla de Eunicea flexuosa y Plexaura kuekenthali (Cnidaria: Octocorallia) en arrecifes coralinos cercanos a fuentes contaminantes en La Habana, Cuba. Serie Oceanológica 11: 91-102.

Ribes, M., Coma, R., GiLi, J.M. 1998. Heterotrophic feeding by gorgonian corals with symbiotic zooxanthella. Limnology and Oceanography 43(6): 1170-1179.

Rogers, C.S., Garrison, G., Grober, R., Hillis, Z.M., Franke, M. A. 2001. Manual para el Monitoreo de Arrecifes de Coral en el Caribe y el Atlántico Occidental. Servicio de Parques Nacionales, Parque Nacional de las Islas Vírgenes.

Rossi, S., Gili, J.M., Garrofé, X. 2011. Net negative growth detected in a population of Leptogorgia sarmentosa: quantifying the biomass loss in a benthic soft bottom gravel gorgonian. Marine Biology 158: 1631-1643.

RowLEY, S.J. 2018. Environmental gradients structure gorgonian assemblages on coral reefs in SE Sulawesi, Indonesia. Coral Reefs 37(2): 609-630.

Sánchez, J.A., Aguilar, C., Dorado, D., Manrique, N. 2007. Phenotypic plasticity and morphological integration in a marine modular invertebrate. BMC Evolutionary Biology 7: $1-9$.

Yeung, C.W., Cheang, C.C., Lee, M.W., Fung, H.L., Chow, W.K., ANG $J_{R}, P$. 2014. Environmental variabilities and the distribution of octocorals and black corals in Hong Kong. Marine Pollution Bulletin 85: 774-782.

Yoshioka, P., Yoshioka, B.B. 1991. A comparison of the survivorship and growth of shallow-water gorgonian species of Puerto Rico. Marine Ecology Progress Series 69: 253-260.

Recibido: 07.03.2018

Aceptado: 18.10.2018 